\title{
Imaging of Endolymphatic Hydrops in Ménière's Disease: A Clinical Update
}

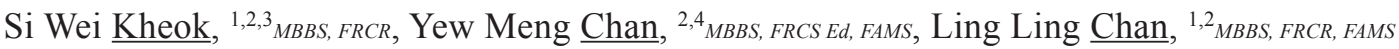

\section{Introduction and Diagnosis of Ménière's Disease}

In 1861, Prosper Ménière described a series of patients with hearing loss and episodic vertigo before the French Academy of Medicine. He linked the condition to inner ear damage. ${ }^{1}$

Since then, Ménière's disease (MD) is known to affect 3.5-513 per 100,000 individuals worldwide. ${ }^{2}$ It is a challenging condition for physicians to diagnose, as patients can have variable presentations. For the unfortunate, it can take years before the diagnosis is established, and hearing loss typically worsens. ${ }^{3}$ Apart from substantial morbidity, there is significant economic cost. The estimated annual loss of earning from MD in the United Kingdom totals GBP442.7 million. ${ }^{4}$

MD is a clinical diagnosis. The clinical classification created in 1995 by the American Academy of Otolaryngology-Head and Neck Surgery Foundation (AAO-HNS), and revised in the 2015 International Classification of Vestibular Disorders by the Bárány Society, include the following two categories: Definite and Probable MD, as defined in Table 1.3,5 Apart from sensorineural hearing loss and episodic vertigo, patients can experience tinnitus and fullness of the affected ear. ${ }^{3,6}$

\section{Aetiopathogenesis and Evolution of MR Imaging}

Endolymphatic hydrops (EH) is a hallmark of MD, which has a complex aetiology that is likely multifactorial. ${ }^{7,8}$ There is propensity for EH to affect the apical turn of the cochlea that can account for low frequency sensorineural hearing loss. Eventually, there is excessive endolymph accumulating in the inner ear, causing damage to the spiral ganglion cells. Some pathology samples show microtears of the Reissner's membrane, ${ }^{7,8}$ leading to postulation that the potassium-rich endolymph escapes and mixes with the perilymph, which is toxic to cochlear hair cells and vestibular sensory neurons of the 8th cranial nerve.

Development of niche magnetic resonance imaging (MRI) techniques to identify endolymphatic hydrops in the clinical setting began in 2007. ${ }^{9}$ Largely driven by Japanese radiologists in the early days,$^{10}$ the technique has been further developed and adopted in hospitals internationally. ${ }^{11,12}$ Prior to this, endolymphatic hydrops

Table 1. Criteria for definite and probable Ménière's disease (MD)

\begin{tabular}{|c|c|}
\hline Definite MD & Probable MD \\
\hline $\begin{array}{l}\text { Two or more spontaneous attacks of vertigo, each lasting } 20 \\
\text { minutes to } 12 \text { hours }\end{array}$ & $\begin{array}{l}\text { - At least } 2 \text { episodes of vertigo or dizziness lasting } 20 \text { minutes } \\
\text { to } 24 \text { hours }\end{array}$ \\
\hline \multirow{3}{*}{$\begin{array}{l}\text { Audiometrically documented fluctuating low- to midfrequency } \\
\text { sensorineural hearing loss in the affected ear on at least } 1 \\
\text { occasion before, during, or after } 1 \text { of the episodes of vertigo } \\
\text { Fluctuating aural symptoms (hearing loss, tinnitus, or fullness) } \\
\text { in the affected ear }\end{array}$} & $\begin{array}{l}\text { - Fluctuating aural symptoms (hearing loss, tinnitus, or fullness) } \\
\text { in the affected ear }\end{array}$ \\
\hline & - Other causes excluded by other tests \\
\hline & \\
\hline - Other causes excluded by other tests & \\
\hline
\end{tabular}

a'Basura GJ, Adams ME, Monfared A, et al. Clinical practice guideline: Ménière’s disease executive summary. Otolaryngol Head Neck Surg 2020;162:415-34.

\footnotetext{
${ }^{1}$ Department of Diagnostic Radiology, Singapore General Hospital, Singapore

${ }^{2}$ Duke-National University of Singapore Graduate Medical School, Singapore

${ }^{3}$ Yong Loo Lin School of Medicine, National University of Singapore, Singapore

${ }^{4}$ Department of Otolaryngology-Head and Neck Surgery, Singapore General Hospital, Singapore

Address for Correspondence: Dr Ling Ling Chan/ Dr Si Wei Kheok, Department of Diagnostic Radiology, Singapore General Hospital, Outram Road, Singapore 169608.

Emails: chan.ling.ling@sgh.com.sg; kheok.si.wei@singhealth.com.sg
} 
was only identified in histopathological specimens and cadaveric studies. ${ }^{7}$

The role of endolymphatic imaging is acknowledged by the European Academy of Otology and Neurotology, although visualisation of EH is not a requirement for the diagnosis of $\mathrm{MD}$, and absence of endolymphatic hydrops does not exclude its diagnosis if the clinical criteria is met. ${ }^{12}$ Other battery of tests available includes audiologic, vestibular assessments, and conventional MRI of the internal auditory meatus to exclude differential diagnosis.

Scientific literature in MRI of EH now includes imaging grading systems and differential diagnosis (Table 2). New imaging evidence bolsters the theories behind the pathoaetiologies of MD.

Table 2. Conditions associated with endolymphatic hydrops

\begin{tabular}{l}
\hline Primary \\
Ménière's disease \\
Vestibular migraine $^{\mathrm{a}}$ \\
Recurrent peripheral vestibulopathy \\
Congenital ear disease (e.g. Mondini dysplasia) ${ }^{\mathrm{c}, \mathrm{d}}$ \\
Secondary $\mathrm{c}, \mathrm{d}$ \\
Vestibular schwannoma \\
Labyrinthitis, meningitis \\
Large vestibular aqueductal syndrome \\
Periductal otosclerosis \\
Trauma and post-surgical (e.g. cochlear implantation, endolymphatic \\
ablation, stapedectomy for otosclerosis) \\
Semicircular dehiscence \\
Others \\
Asymptomatic
\end{tabular}

${ }^{a}$ Gürkov R, Kantner C, Strupp M, et al. Endolymphatic hydrops in patients with vestibular migraine and auditory symptoms. Eur Arch Otorhinolaryngol 2014;271:2661-7.

${ }^{\mathrm{b}}$ Attyé A, Dumas G, Troprès I, et al. Recurrent peripheral vestibulopathy: Is MRI useful for the diagnosis of endolymphatic hydrops in clinical practice? Eur Radiol 2015;25:3043-9.

c Ferster A, Cureoglu S, Keskin N, et al. Secondary endolymphatic hydrops. Otol Neurotol 2017;38:774-9.

d Gürkov R, Pyykö I, Zou J, et al. What is Menière's disease? A contemporary re-evaluation of endolymphatic hydrops. J Neurol 2016;263:71-81.

${ }^{e}$ Nakashima T, Sone M, Teranishi M, et al. A perspective from magnetic resonance imaging findings of the inner ear: Relationships among cerebrospinal, ocular and inner ear fluids. Auris Nasus Larynx 2012;39:345-55.

\section{The Role of Electrophysiological Tests in the Diagnosis of Ménière's Disease}

Current diagnostic workup of MD relies on serial audiometric changes in pure tone audiometry or speech discrimination scores in relation to a vertiginous attack. Additional neurophysiological tests have attendant limitations and results need to be interpreted with caution. ${ }^{13,14}$

Dehydration tests using glycerol and frusemide reduce endolymphatic volume and pressure, but further audio-vestibular tests are required. Significant hearing threshold improvement was evident in $31 \%$ of 32 patients $(10 \mathrm{~dB}$ or more at 2 frequencies or $12 \%$ speech discrimination improvement). ${ }^{15}$ Another study found $53 \%$ hearing improvement following a dehydration test, with 2 of the unaffected ears showing positive glycerol test. ${ }^{16}$ Standards to compare and determine the auditory thresholds may be prone to errors, more so with the fluctuating hearing pattern of MD.

Including tests such as vestibular evoked myogenic potential (VEMP) to document interval improvement, may add to cost. ${ }^{13,17}$ Sensitivity of dehydration tests varies as the disease fluctuates and progresses, positive pick-up being higher in early stages but lower in remission and advanced stages. Although cervical and ocular VEMPs offer objective quantitative measures of otolith functions relating to the saccule and utricle respectively, recent practice guidelines from the American Academy of Neurology concluded that there is inconclusive evidence whether VEMPs reliably diagnose MD..$^{14,18}$

Electronystagmography, a neurophysiological test of the lateral semicircular canal based on the vestibularocular reflex, may support the diagnosis of MD when peripheral weakness is found on caloric testing in the presence of hearing loss and normal video head impulse test findings. ${ }^{13}$

However, this is not perfect, as Casani describes normal caloric responses from $9-29 \%$ of his study population with unilateral Definite MD at various stages of hearing loss, and from $100 \%$ of patients with canal paresis when the loss is greater than $70 \mathrm{~dB} .{ }^{19}$ Furthermore, a valid objection to caloric testing is the aggravation of vertigo in patients with MD.

Electrocochleography uses extratympanic or invasive intratympanic electrodes to record electrical potentials generated in the auditory nerve (AP) and the cochlear summating potential (SP) after a sound stimulus. Enlarged SP/AP amplitude ratios correlate with expanded endolymphatic volume in cochlear $\mathrm{EH}$, but 
again, fluctuating symptoms limit their applicability as a diagnostic tool in the early course of disease. ${ }^{14,20}$

Fukuoka et al. compared MRI, electrocochleography and the glycerol dehydration test in 20 patients diagnosed with definite MD. The latter two techniques yielded positive results in 11 and 12 patients, respectively, and in 15 patients overall on at least one of the two neurophysiological tests. In comparison, MRI was positive for hydrops in 19 patients. ${ }^{21}$

In another paper, Laureline Kahn et al. performed a retrospective study of 31 definite MD patients who were imaged with 3D fluid-attenuated inversion recovery (FLAIR) MRI sequence. They reported no significant correlation between the presence of saccular hydrops versus cervical VEMP, utricular hydrops versus ocular VEMP, and ampullar hydrops versus video head impulse test. However, the severity of endolymphatic hydrops on MRI was correlated with the degree of hearing loss. ${ }^{22}$

\section{MRI Technicalities and Radiological Assessment}

Current state-of-the-art techniques for imaging of MD require gadolinium-based contrast medium to be introduced into the perilymph either via the intravenous or trans-tympanic routes. Initial studies were carried out via trans-tympanic contrast administration. ${ }^{10}$ The advantage of this mode is the smaller dose of diluted contrast locally introduced $(<0.1 \%$ ordinarily given via intravenous route), ${ }^{23}$ reducing systemic contrast exposure. This method has the dual advantage of concurrent assessment of the feasibility of transtympanic gentamicin therapy for $\mathrm{MD}$, with regards to access to the entire membranous labyrinth. However, this is invasive with tympanic membrane puncture, and requires additional radio-otological coordination for imaging 12 to 24 hours later, when the contrast reaches the perilymph in the entire labyrinth. ${ }^{9,10,24}$ There are also logistical challenges with tight MR scheduling.

The intravenous route is less invasive and more convenient for the patient as MR imaging is performed only 4 hours after contrast injection. ${ }^{25}$ In addition, both ears are assessed simultaneously after a single intravenous injection, whereas the trans-tympanic route requires separate punctures when both sides are to be assessed. If the scan is meant to assess for diseases associated with $\mathrm{EH}$ by destruction of the stria vascularis, such as circulatory disturbances and trauma, the intravenous route is also more suited..$^{23}$

Contrast causes the perilymph in the scala vestibuli and tympani to be enhanced. Due to the intact endolymph-blood barrier, the endolymph in the scala media does not enhance, appearing dark (hypointense) against the bright (hyperintense) perilymph within the labyrinth on the MR images (Figs. 1 and 2).

Several MRI sequences have been developed to identify EH. Two commonly used ones are 3D FLAIR, and 3D Inversion Recovery with real reconstruction (3D real IR). 3D FLAIR is more sensitive than T1-weighted imaging to faint gadolinium enhancement. Moreover, heavily T2-weighted 3D FLAIR with a long effective echo time specifically heightens sensitivity to low gadolinium concentrations, enabling the use of single-dose intravenous contrast

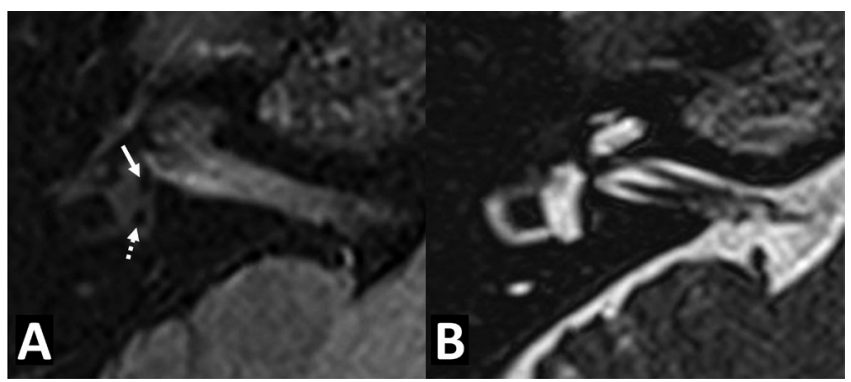

Fig. 1. Axial (A) 3D real inversion recovery post-contrast and (B) cisternographic constructive interference in steady state (CISS) images of the healthy ear show normal-size saccule (white arrow) and utricle (white-dashed arrow) as small black dots within the bony vestibule, yielding saccule to utricle area ratio inversion (SURI) $<1$ score. The perilymph is mildly enhancing and there is no endolymphatic hydrops.

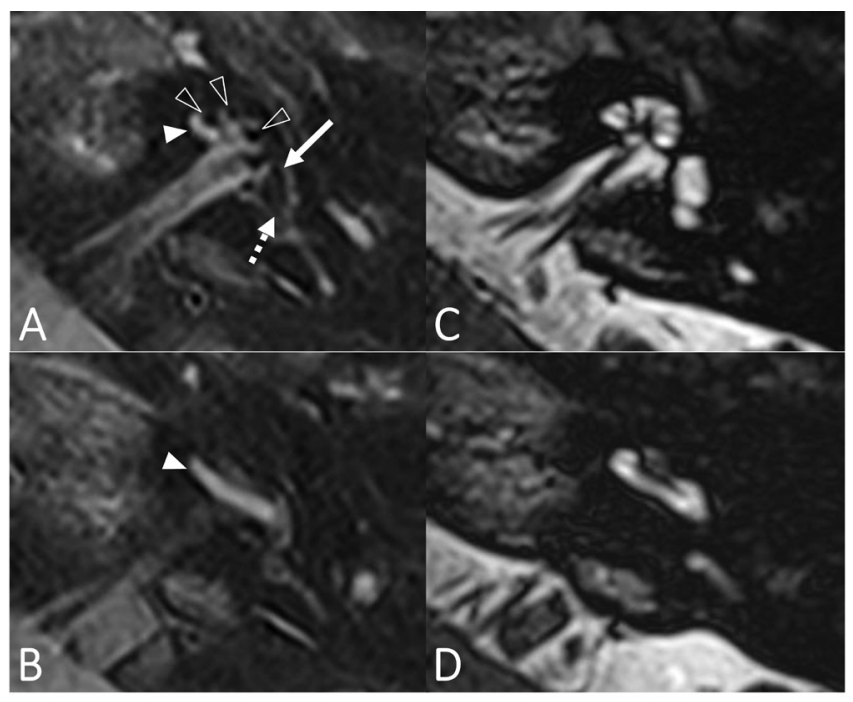

Fig. 2. Axial (A, B) 3D real inversion recovery post-contrast and (C, D) cisternographic constructive interference in steady state (CISS) images of the diseased ear in a patient with left-sided Ménière's disease. The distended hypointense saccule (arrow) nearly fills the entire bony vestibule, and is larger than the utricle (dashed arrow), yielding a grading of SURI $>1$. Thin enhancing rim of perilymph is still visible, congruent with grade 1 vestibular hydrops by Barath grading. Abnormally prominent perilymph enhancement in the cochlea (solid arrowheads) well depicts the non-enhancing, hypointense, distended cochlear duct (open arrowheads) that completely obstructs the scala vestibuli, compatible with grade 2 cochlear hydrops. 
injection. ${ }^{26}$ It is noteworthy that shortening inversion times in 3D FLAIR can suppress the gadolinium signal in the perilymph and produce endolymph hyperintense images instead, allowing for semiquantitative assessments using endolymph: perilymph ratio. However, reproducibility is sensitive to optimal inversion time selection. ${ }^{18,19}$ Various image post-processing techniques - through fusion by image subtraction or multiplication involving MR cisternographic images and heavily T2-weighted 3D FLAIR images-enhance the contrast-to-noise ratio between the endolymph, perilymph and bone. ${ }^{27,28}$ However, 3D real inversion recovery using phase-sensitive reconstructions to delineate the EH boundary from the surrounding bone and air suffices, and precludes the need for additional image processing and mis-registration pitfalls..$^{10,27}$

At least 7 different imaging grading systems are available, and we summarise 4 that we use clinically in Table $3 .^{29-32}$ Most of these analyse the degree that the EH bulges into the scala vestibuli, similar to pathological grading employed. ${ }^{7}$ Others analyse volumetry, proximity to the round window, and the saccule-utricle comparison. ${ }^{32,33}$

\section{Radiological Findings}

Concurring with pathological findings, in vivo MRI EH study demonstrates the tendency for severe EH to occur in the symptomatic ear. ${ }^{7}$ Findings of severe EH (grade 2) on imaging are more specific for Ménière's disease (Table 3, Nagoya Scale), ${ }_{11}^{1}$ while mild hydrops can be present in clinically asymptomatic ears, and are as yet of uncertain clinical significance. ${ }^{711}$ Evidently, the longer the duration of MD, the more marked is the EH. ${ }^{11}$ In addition, vestibular hydrops is also a more distinctive primary imaging finding in MD than cochlear hydrops. ${ }^{16}$

The saccule to utricle area ratio inversion (SURI) classification is founded on the fact that the saccule is smaller than the utricle in a healthy ear, ${ }^{34}$ whereas the saccule is often dilated compared to the utricle in MD. ${ }^{7,32}$ Understandably, the SURI classification is not applicable when the saccule and utricle appear fused in the scan. Additionally, if the saccule is not visualised in a patient with $\mathrm{MD}$, it has been postulated that the saccule has collapsed or ruptured/fistulised, ${ }^{11,35}$ and enhancement of the endolymphatic duct in such a case will support the premise of a ruptured Reissner's membrane..$^{23,36}$

Greater perilymph enhancement secondary to bloodlabyrinth barrier breakdown is associated with the pathological ear in MD, a higher functional level on audiologic tests at the time of MR assessment than those without breakdown, and duration of disease. ${ }^{30,33}$

The imaging findings also need to be interpreted in correlation with the clinical picture (Table 2), given that not all differentials for endolymphatic hydrops can be diagnosed radiologically, and mild hydrops are reported in asymptomatic individuals.

\section{Clinical Impact}

The earlier AAO-HNS 1995 guideline for MD included the definition of "certain MD" that was removed in the 2015 guidelines. ${ }^{5}$ This removal referred to its need for histopathologic confirmation, which is now deemed of little clinical utility since it entailed surgical resection or autopsy. Furthermore, while MRI of EH was unavailable in 1995, it is accessible in specialised imaging centres today.

MRI endolymphatic hydrops may facilitate earlier diagnosis for MD, and is part of the clinician's armamentarium in evaluating patients with profound hearing loss, when functional tests such as electrocochleography and glycerol test cannot be reliably used. ${ }^{23}$

Endolymphatic hydrops imaging with intratympanically administered contrast may also be used in assessing the suitability of trans-tympanic treatment. ${ }^{9}$ Prospective MRI studies to determine if patients with unilateral disease and bilateral endolymphatic hydrops are susceptible to developing symptoms in the asymptomatic ear will deepen our understanding of inner ear pathologies. The definitive role of $\mathrm{EH}$ imaging in the evaluation of related disorders such as recurrent peripheral vestibulopathy, vestibular migraine or sudden deafness remains to be determined in further clinical studies. $^{24,25}$

EH imaging is performed in tandem with the conventional internal acoustic meatus MRI. The latter excludes important differential diagnosis of endolymphatic hydrops in the imaging diagnostic workup of patients with MD presentation (Table 2). Naturally, this increases the total duration of the study and entails additional cost. Thus, appropriate patient counselling and engagement for this workup also need attention.

Hospitals and radiology departments with interest in this area need to develop a workflow, fine-tune the MRI sequences on their systems that meet clinical needs, and organise a validated clinical pipeline for radiological reporting. Dedicated radiologists familiar with the delicate anatomy of the temporal bone structures and differentials of MD and EH are critical. Continual 


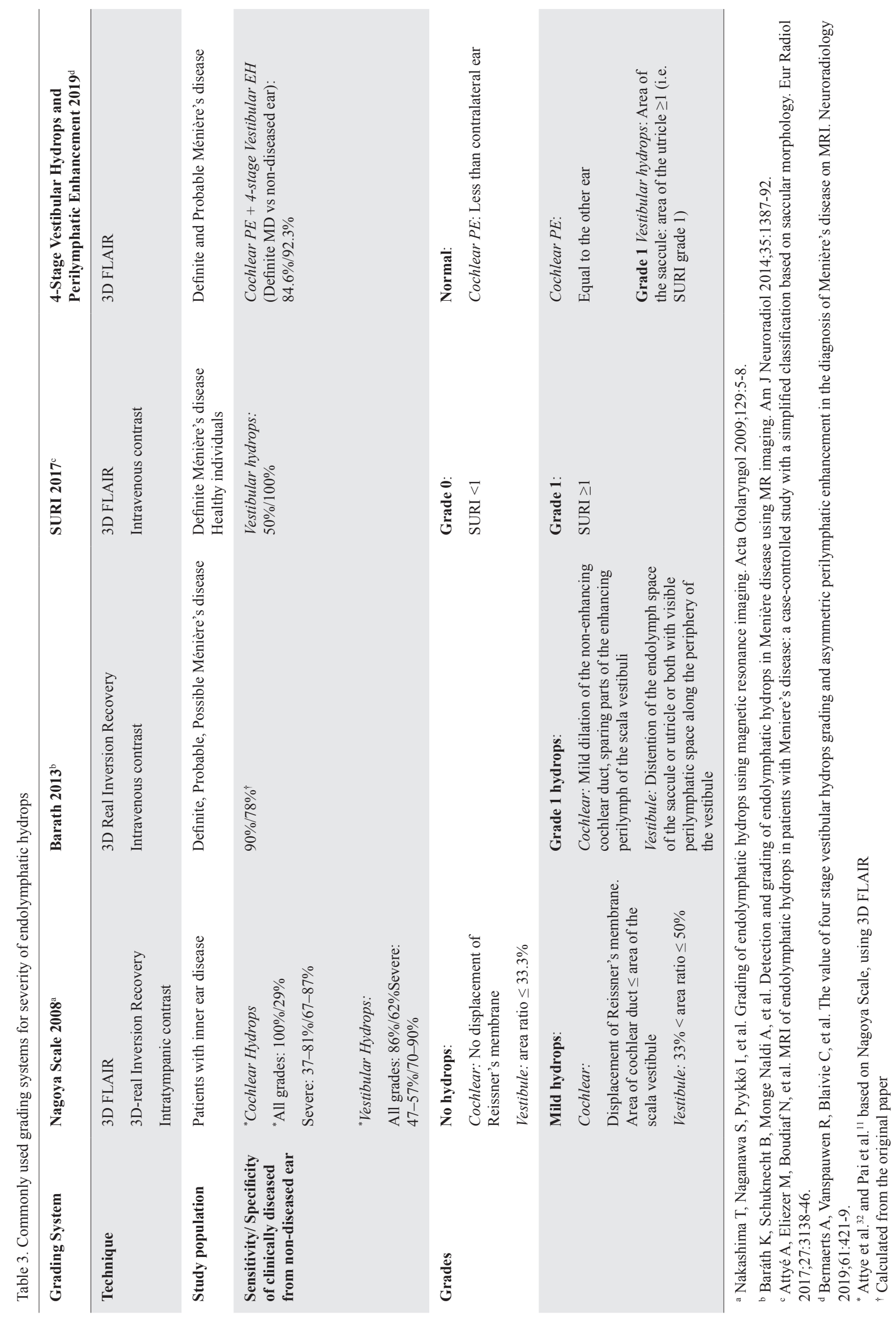




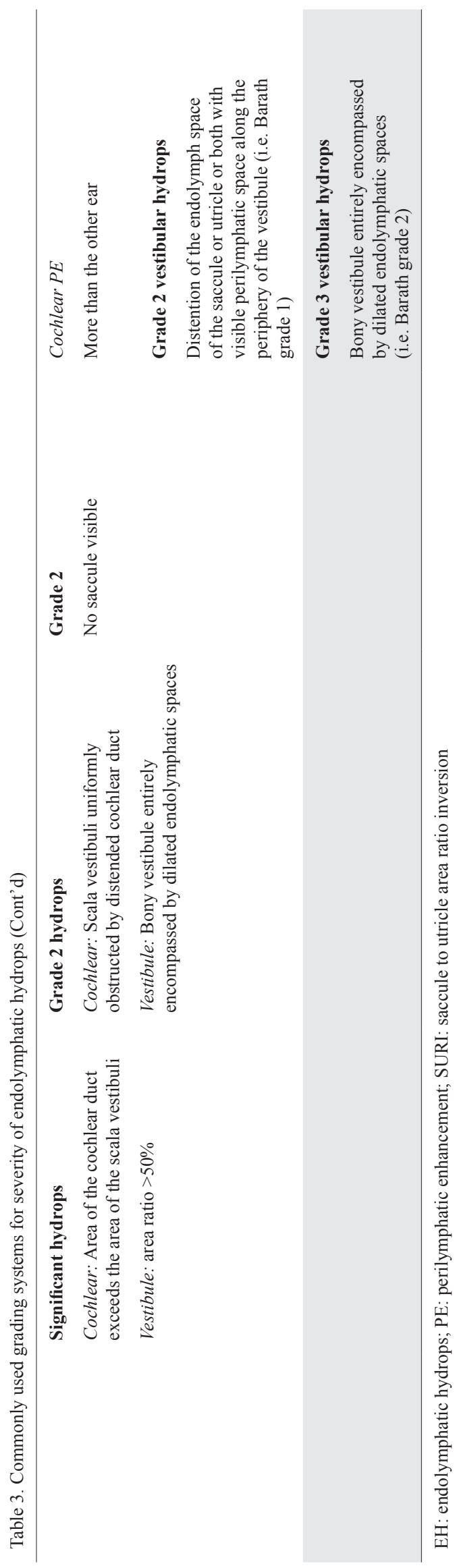

clinico-radiological engagement between radiologists and experienced ENT or neurology colleagues with expertise in assessment of vestibulopathy is crucial in improving patient management.

\section{Future Directions}

Using a 3D T2-weighted steady state free precession sequence to imaging MD obviates the need for contrast administration to enhance the perilymph for depiction of the $\mathrm{EH}$ altogether. ${ }^{37,38}$ This proposes direct visualisation of the saccule for identification of its morphological changes; however, validation and reproducibility assessments await verification. ${ }^{39}$ Further improvement in spatial resolution that could be achieved on ultra-high field MR imaging at 7 Tesla systems offers hope for improved linear quantification of fine intra-labyrinthine structures and lateralisation of the symptomatic ear. In addition, as demand for MR imaging in the diagnostic workup of MD and $\mathrm{EH}$ rises, deep-learning techniques also show promise in rapid, automated analysis. ${ }^{40}$

We have reviewed the evolution and clinical implementation of specialised state-of-the-art highresolution MR techniques to identify $\mathrm{EH}$ in MD. Engaging the relevant radiological and clinical teams is of paramount importance to translate these novel MR imaging into clinical practice and impact patient outcome.

\section{REFERENCES}

1. Baloh RW. Prosper Ménière and his disease. Arch Neurol 2001;58:1151-6.

2. Harris JP, Alexander TH. Current-day prevalence of Ménière's syndrome. Audiol Neurotol 2010;15:318-22.

3. Basura GJ, Adams ME, Monfared A, et al. Clinical practice guideline: Ménière's disease executive summary. Otolaryngol Head Neck Surg 2020;162:415-34.

4. Tyrrell J, Whinney DJ, Taylor T. The cost of Ménière's disease: a novel multisource approach. Ear Hear 2016;37:e202-9.

5. Goebel JA. 2015 Equilibrium committee amendment to the 1995 AAO-HNS guidelines for the definition of Ménière's disease. Otolaryngol Head Neck Surg 2016;154:403-4.

6. Shea JJ. Medical and surgical treatment of Meniere's disease. Ann Acad Med Singap 1991;20:686-9.

7. Sperling NM, Paparella MM, Yoon TH, et al. Symptomatic versus asymptomatic endolymphatic hydrops. Laryngoscope 1993;103:277-85.

8. Schuknecht HF. Meniere's disease: a correlation of symptomatology and pathology. Laryngoscope 1963;73:651-65.

9. Nakashima T, Naganawa S, Sugiura M, et al. Visualization of endolymphatic hydrops in patients with Meniere's disease. Laryngoscope 2007;117:415-20.

10. Naganawa S, Satake H, Kawamura M, et al. Separate visualization of endolymphatic space, perilymphatic space and bone by a single pulse sequence; $3 \mathrm{D}$-inversion recovery imaging utilizing real reconstruction 
after intratympanic Gd-DTPA administration at 3 Tesla. Eur Radiol 2008;18:920-4.

11. Pai I, Mendis S, Murdin L, et al. Magnetic resonance imaging of Ménière's disease: early clinical experience in a UK centre. J Laryngol Otol 2020;134:302-310.

12. Magnan J, Özgirgin ON, Trabalzini F, et al. European position statement on diagnosis, and treatment of Meniere's disease. J Int Adv Otol 2018;14:317-21.

13. Güneri EA, Çakir A, Mutlu B. Validity and reliability of the diagnostic tests for Ménière's disease. Turk Arch Otolaryngol 2016; 54:124-30.

14. Ciorba A, Skarżyński PH, Corazzi V, et al. Assessment tools for use in patients with Ménière disease: Turk An update. Med Sci Monit 2017;23:6144-9.

15. Lee JD, Kim HJ, Jung J, et al. Is dehydration test using isosorbide useful in Meniere's disease? Acta Otolaryngol 2016;136:1107-9.

16. Mohamed ES, Said EA, Mahmoud NA. Effect of glycerol test on audiovestibular tests in patients with Meniere's disease. Egypt J Ear, Nose, Throat Allied Sc 2017;18:115-20.

17. Seo T, Shiraishi K, Kobayashi T, et al. Revision of a furosemideloading vestibular-evoked myogenic potential protocol for detecting endolymphatic hydrops. Acta Otolaryngol 2017;137:1244-8.

18. Fife TD, Colebatch JG, Kerber KA, et al. Practice guideline: Cervical and ocular vestibular evokedmyogenic potential testing: Report of the guideline development, dissemination, and implementation subcommittee of the American Academy of Neurology. Neurology 2017;89:2288-96.

19. Cerchiai N, Navari E, Miccoli M, et al. Menière's disease and caloric stimulation: some News from an old test. J Int Adv Otol 2019;15:442-6.

20. Lamounier P, Gobbo DA, de Souza TSA, et al. Electrocochleography for Ménière's disease: Is it reliable? Braz J Otorhinolaryngol 2014;80:527-32.

21. Fukuoka H, Takumi Y, Tsukada K, et al. Comparison of the diagnostic value of 3 T MRI after intratympanic injection of GBCA, electrocochleography, and the glycerol test in patients with Meniere's disease. Acta Otolaryngol 2012;132:141-5.

22. Kahn L, Hautefort C, Guichard JP, et al. Relationship between video head impulse test, ocular and cervical vestibular evoked myogenic potentials, and compartmental magnetic resonance imaging classification in Menière's disease. Laryngoscope 2020;130:E444-52.

23. Pyykkö I, Zou J, Poe D, et al. Magnetic resonance imaging of the inner ear in Meniere's disease. Otolaryngol Clin North Am 2010;43:1059-80.

24. Zou J, Pyykkö I, Bjelke B, et al. Communication between the perilymphatic scalae and spiral ligament visualized by in vivo MRI. Audiol Neurotol 2005; 10:145-52.

25. Naganawa S, Yamazaki M, Kawai H, et al. Imaging of endolymphatic and perilymphatic fluid after intravenous administration of singledose gadodiamide. Magn Reson Med Sci 2012;11:145-50

26. Naganawa S, Yamazaki M, Kawai H, et al. Visualization of endolymphatic hydrops in Ménière's disease with single-dose intravenous gadolinium-based contrast media using heavily T2-weighted 3D-FLAIR. Magn Reson Med Sci 2010;9:237-42.

27. Ohashi T, Naganawa S, Takeuchi A, et al. Quantification of endolymphatic space volume after intravenous administration of a single dose of gadolinium-based contrast agent: 3D-real inversion recovery versus HYDROPS-Mi2. Magn Reson Med Sci 2020;19:119-24.

28. Naganawa S, Yamazaki M, Kawai H, et al. Imaging of Ménière's disease by subtraction of MR cisternography from positive perilymph image. Magn Reson Med Sci 2012;11:303-9.

29. Gürkov R, Barath K, de Foer B, et al. A plea for systematic literature analysis and conclusive study design, comment on: "Systematic review of magnetic resonance imaging for diagnosis of Meniere disease". J Vestib Res 2019;1-7.

30. Bernaerts A, Vanspauwen R, Blaivie C, et al. The value of four stage vestibular hydrops grading and asymmetric perilymphatic enhancement in the diagnosis of Menière's disease on MRI. Neuroradiology 2019;61:421-9.

31. Baráth K, Schuknecht B, Monge Naldi A, et al. Detection and grading of endolymphatic hydrops in Menière disease using MR imaging. Am J Neuroradiol 2014;35:1387-92.

32. Attyé A, Eliezer M, Boudiaf N, et al. MRI of endolymphatic hydrops in patients with Meniere's disease: a case-controlled study with a simplified classification based on saccular morphology. Eur Radiol 2017;27:3138-46.

33. Conte $\mathrm{G}$, Caschera L, Calloni $\mathrm{S}$, et al. MR imaging in Menière disease: Is the contact between the vestibular endolymphatic space and the oval window a reliable biomarker? Am J Neuroradiol 2018;39:2114-9.

34. Morita N, Kariya S, Deroee AF, et al. Membranous labyrinth volumes in normal ears and Ménière disease: A three-dimensional reconstruction study. Laryngoscope 2009;119:2216-20.

35. Lopez-Escamez JA, Attyé A. Systematic review of magnetic resonance imaging for diagnosis of Meniere disease. J Vestib Res Equilib Orientat 2019;29:121-9.

36. Nakashima T, Naganawa S, Pyykkö I, et al. Grading of endolymphatic hydrops using magnetic resonance imaging. Acta Otolaryngol 2009;129:5-8

37. Venkatasamy A, Veillon F, Fleury A, et al. Imaging of the saccule for the diagnosis of endolymphatic hydrops in Meniere disease, using a three-dimensional T2-weighted steady state free precession sequence: accurate, fast, and without contrast material intravenous injection. Eur Radiol Exp 2017;1:1-8.

38. Simon F, Guichard JP, Kania R, et al. Saccular measurements in routine MRI can predict hydrops in Menière's disease. Eur Arch Oto-Rhino-Laryngology 2017;274:4113-20.

39. Dominguez $\mathrm{P}$, Naganawa $\mathrm{S}$. Letter to the editor on the article "Saccular measurements in routine MRI can predict hydrops in Ménière's disease" by Simon F et al. Eur Arch Otorhinolaryngol 2018;275:311-2

40. Cho YS, Cho K, Park CJ, et al. Automated measurement of hydrops ratio from MRI in patients with Ménière's disease using CNN-based segmentation. Sci Rep 2020;10:1-10 